\title{
Естественные $p$-логики
}

\author{
H. E. TомовА
}

\begin{abstract}
In this paper the notion of $p$-logic is generalied and the class of natural $p$-logics and it's functional properties are considered. It is shown that paraconsistent logic $\mathbf{P}^{\mathbf{1}}$ and paracomplete logic $\mathbf{I}^{\mathbf{1}}$ are funclionally equivalent. In conclusion natural $p$-logics are presented as a lattice.

Ключевые слова: трехзначные логики, $p$-логики, естественные $p$ логики, решетка естественных $p$-логик, функциональные свойства $p$-логик
\end{abstract}

В работе [3] по аналогии с понятием $p$-алгебры и дважды $p$ алгебры вводится понятие $p$-логики и дважды $p$-логики. $P$-логика задается связками $\vee, \wedge$,$\rceil , дуальная p$-логика - связками $\vee, \wedge,\lceil. \vee$ и $\wedge$ есть обычные $\max ($ дизъюнкция) и $\min ($ конъюнкция), а $\rceil$ и $\lceil$ задаются следющими таблицами:

\begin{tabular}{|c|c|c|}
\hline$p$ & \rceil $p$ & $\lceil p$ \\
\hline 1 & 0 & 0 \\
$\frac{1}{2}$ & 0 & 1 \\
0 & 1 & 1 \\
\hline
\end{tabular}

Логика со связками $\vee, \wedge$,$\rceil и \lceil$ есть дважды $p$-логика.

В данной статье обобщается понятие $p$-логики и рассматривается класс естественных $p$-логик, подробно исследуются функциональные свойства этого класса систем.

При определении класса естественных $p$-логик ключевую роль играет заданный нами в [4] класс естественных импликаций.

Посредством импликации ${ }^{1}$ следующим образом определим дизъюнкцию:

$$
x \vee y={ }_{D f}\left(x \rightarrow_{i} y\right) \rightarrow_{i} y(1 \leq i \leq 28) .
$$

\footnotetext{
${ }^{1}$ Здесь и далее импликации с указанными номерами см. в [4].
} 
В результате получаем 18 уникальных дизъюнкций. Далее определим двойственную связку - конъюнкцию:

$$
x \wedge^{i} y={ }_{D f} \sim\left(\sim x \vee^{i} \sim y\right)(1 \leq i \leq 18) .
$$

Таким образом, имеем 18 естественных $p$-логик (логик со связками $\left.\left\{\vee^{i}, \wedge^{i},\right\rceil\right\}$, где $\left.(1 \leq i \leq 18)\right)$ и соответственно 18 естественных дуальных $p$-логик (логик со связками $\left\{\vee^{i}, \wedge^{i},\lceil\}\right.$, где $(1 \leq i \leq 18))$. Приведем таблицы истинности, соответствующие дизъюнкциям и конъюнкциям естественных $p$-логик, и перенумеруем их.

\begin{tabular}{|c|c|c|c|c|c|c|c|c|c|c|c|c|c|c|c|}
\hline $\mathrm{V}^{1}$ & 1 & $\frac{1}{2}$ & 0 & $\wedge^{1}$ & 1 & $\frac{1}{2}$ & 0 & $V^{2}$ & 1 & $\frac{1}{2}$ & 0 & $\wedge^{2}$ & 1 & $\frac{1}{2}$ & 0 \\
\hline 1 & 1 & 1 & 1 & 1 & 1 & $\frac{1}{2}$ & 0 & 1 & 1 & 1 & 1 & 1 & 1 & $\frac{1}{2}$ & 0 \\
\hline$\frac{1}{2}$ & 1 & $\frac{1}{2}$ & 1 & $\frac{1}{2}$ & 0 & $\frac{1}{2}$ & 0 & $\frac{1}{2}$ & 1 & $\frac{1}{2}$ & 0 & $\frac{1}{2}$ & 0 & $\frac{1}{2}$ & 0 \\
\hline 0 & 1 & $\frac{1}{2}$ & 0 & 0 & 0 & 0 & 0 & 0 & 1 & $\frac{1}{2}$ & 0 & 0 & 0 & 0 & 0 \\
\hline
\end{tabular}

\begin{tabular}{|c|c|c|c|c|c|c|c|c|c|c|c|c|c|c|c|}
\hline$v^{3}$ & 1 & $\frac{1}{2}$ & 0 & $\wedge^{3}$ & 1 & 2 & 0 & $\mathrm{~V}^{4}$ & 1 & $\frac{1}{2}$ & 0 & $\wedge^{4}$ & 1 & $\frac{1}{2}$ & 0 \\
\hline 1 & 1 & 1 & 1 & 1 & 1 & $\frac{1}{2}$ & 0 & 1 & 1 & 1 & 1 & 1 & 1 & 1 & 0 \\
\hline$\frac{1}{2}$ & 1 & $\frac{1}{2}$ & $\frac{1}{2}$ & $\frac{1}{2}$ & $\frac{1}{2}$ & $\overline{2}$ & 0 & $\frac{1}{2}$ & 1 & 0 & 1 & $\frac{1}{2}$ & 0 & 1 & 0 \\
\hline 0 & 1 & $\frac{1}{2}$ & 0 & 0 & 0 & 0 & 0 & 0 & 1 & 0 & 0 & 0 & 0 & 0 & 0 \\
\hline
\end{tabular}

\begin{tabular}{|c|c|c|c|c|c|c|c|c|c|c|c|c|c|c|c|}
\hline$\vee^{5}$ & 1 & $\frac{1}{2}$ & 0 & $\wedge^{5}$ & 1 & $\frac{1}{2}$ & 0 & $\vee^{6}$ & 1 & $\frac{1}{2}$ & 0 & $\wedge^{6}$ & 1 & $\frac{1}{2}$ & 0 \\
\hline 1 & 1 & 1 & 1 & 1 & 1 & 1 & 0 & 1 & 1 & 1 & 1 & 1 & 1 & 1 & 0 \\
\hline$\frac{1}{2}$ & 1 & 0 & 0 & $\frac{1}{2}$ & 1 & 1 & 0 & $\frac{1}{2}$ & 1 & 0 & $\frac{1}{2}$ & $\frac{1}{2}$ & $\frac{1}{2}$ & 1 & 0 \\
\hline 0 & 1 & 0 & 0 & 0 & 0 & 0 & 0 & 0 & 1 & 0 & 0 & 0 & 0 & 0 & 0 \\
\hline
\end{tabular}

\begin{tabular}{|c|c|c|c|c|c|c|c|c|c|c|c|c|c|c|c|}
\hline$V^{7}$ & 1 & $\frac{1}{2}$ & 0 & $\wedge^{7}$ & 1 & $\frac{1}{2}$ & 0 & $V^{8}$ & 1 & $\frac{1}{2}$ & 0 & $\wedge^{8}$ & 1 & $\frac{1}{2}$ & 0 \\
\hline 1 & 1 & 1 & 1 & 1 & 1 & 0 & 0 & 1 & 1 & 1 & 1 & 1 & $\overline{2}$ & 0 & 0 \\
\hline$\frac{1}{2}$ & 1 & 1 & 1 & $\frac{1}{2}$ & 0 & 0 & 0 & $\frac{1}{2}$ & $\frac{1}{2}$ & 1 & 1 & $\frac{1}{2}$ & 0 & 0 & $\frac{1}{2}$ \\
\hline 0 & 1 & 1 & 0 & 0 & 0 & 0 & 0 & 0 & 1 & 1 & 0 & 0 & 0 & 0 & 0 \\
\hline
\end{tabular}

\begin{tabular}{|c|c|c|c|c|c|c|c|c|c|c|c|c|c|c|c|}
\hline $\mathrm{V}^{9}$ & 1 & $\frac{1}{2}$ & 0 & $\wedge^{9}$ & 1 & $\frac{1}{2}$ & 0 & $\vee^{10}$ & 1 & $\frac{1}{2}$ & 0 & $\wedge^{10}$ & 1 & $\frac{1}{2}$ & 0 \\
\hline 1 & 1 & 1 & 1 & 1 & 1 & $\frac{1}{2}$ & 0 & 1 & 1 & 1 & 1 & 1 & 1 & 0 & 0 \\
\hline$\frac{1}{2}$ & $\frac{1}{2}$ & $\frac{1}{2}$ & 1 & $\frac{1}{2}$ & 0 & $\frac{1}{2}$ & $\frac{1}{2}$ & $\frac{1}{2}$ & $\frac{1}{2}$ & $\frac{1}{2}$ & 1 & $\frac{1}{2}$ & 0 & $\frac{1}{2}$ & $\frac{1}{2}$ \\
\hline 0 & 1 & $\frac{1}{2}$ & 0 & 0 & 0 & 0 & 0 & 0 & 1 & 1 & 0 & 0 & 0 & 0 & 0 \\
\hline
\end{tabular}




\begin{tabular}{|c|c|c|c|c|c|c|c|c|c|c|c|c|c|c|c|}
\hline$V^{11}$ & 1 & $\frac{1}{2}$ & 0 & $\wedge^{11}$ & 1 & $\frac{1}{2}$ & 0 & $\vee^{12}$ & 1 & $\overline{2}$ & 0 & $\wedge^{12}$ & 1 & $\frac{1}{2}$ & 0 \\
\hline 1 & 1 & 1 & 1 & 1 & 1 & 0 & 0 & 1 & 1 & 1 & 1 & 1 & 1 & 1 & 0 \\
\hline$\frac{1}{2}$ & 1 & $\frac{1}{2}$ & 1 & $\frac{1}{2}$ & 0 & $\frac{1}{2}$ & 0 & $\frac{1}{2}$ & $\frac{1}{2}$ & 0 & 1 & $\frac{1}{2}$ & 0 & 1 & $\overline{2}$ \\
\hline 0 & 1 & 1 & 0 & 0 & 0 & 0 & 0 & 0 & 1 & 0 & 0 & 0 & 0 & 0 & 0 \\
\hline
\end{tabular}

\begin{tabular}{|c|c|c|c|c|c|c|c|c|c|c|c|c|c|c|c|}
\hline$\vee^{13}$ & 1 & $\frac{1}{2}$ & 0 & $\wedge^{13}$ & 1 & $\frac{1}{2}$ & 0 & $\bigvee^{14}$ & 1 & $\frac{1}{2}$ & 0 & $\wedge^{14}$ & 1 & $\frac{1}{2}$ & 0 \\
\hline 1 & 1 & $\frac{1}{2}$ & 1 & 1 & 1 & 0 & 0 & 1 & 1 & $\frac{1}{2}$ & 1 & 1 & 1 & 0 & 0 \\
\hline$\frac{1}{2}$ & 1 & 0 & 1 & $\frac{1}{2}$ & 0 & 1 & 0 & $\frac{1}{2}$ & $\frac{1}{2}$ & 0 & 1 & $\frac{1}{2}$ & 0 & 1 & $\frac{1}{2}$ \\
\hline 0 & 1 & 1 & 0 & 0 & 0 & $\frac{1}{2}$ & 0 & 0 & 1 & 1 & 0 & 0 & 0 & $\frac{1}{2}$ & 0 \\
\hline
\end{tabular}

\begin{tabular}{|c|c|c|c|c|c|c|c|c|c|c|c|c|c|c|c|}
\hline$\vee^{15}$ & 1 & $\frac{1}{2}$ & 0 & $\wedge^{15}$ & 1 & $\frac{1}{2}$ & 0 & $\vee^{16}$ & 1 & $\frac{1}{2}$ & 0 & $\wedge^{16}$ & 1 & $\frac{1}{2}$ & 0 \\
\hline 1 & 1 & $\frac{1}{2}$ & 1 & 1 & 1 & $\frac{1}{2}$ & 0 & 1 & 1 & $\frac{1}{2}$ & 1 & 1 & 1 & $\frac{1}{2}$ & 0 \\
\hline$\frac{1}{2}$ & 1 & $\frac{1}{2}$ & 1 & $\frac{1}{2}$ & 0 & $\frac{1}{2}$ & 0 & $\frac{1}{2}$ & $\frac{1}{2}$ & $\frac{1}{2}$ & 1 & $\frac{1}{2}$ & 0 & $\frac{1}{2}$ & $\frac{1}{2}$ \\
\hline 0 & 1 & $\frac{1}{2}$ & 0 & 0 & 0 & $\frac{1}{2}$ & 0 & 0 & 1 & $\frac{1}{2}$ & 0 & 0 & 0 & $\frac{1}{2}$ & 0 \\
\hline
\end{tabular}

\begin{tabular}{|c|c|c|c|c|c|c|c|c|c|c|c|c|c|c|c|}
\hline$\vee^{17}$ & 1 & $\frac{1}{2}$ & 0 & $\wedge^{17}$ & 1 & $\frac{1}{2}$ & 0 & $\vee^{18}$ & 1 & $\frac{1}{2}$ & 0 & $\wedge^{18}$ & 1 & $\frac{1}{2}$ & 0 \\
\hline 1 & 1 & 1 & 1 & 1 & 1 & 1 & 0 & 1 & 1 & 1 & 1 & 1 & 1 & 1 & 0 \\
\hline$\frac{1}{2}$ & 1 & $\frac{1}{2}$ & 1 & $\frac{1}{2}$ & 0 & $\frac{1}{2}$ & 0 & $\frac{1}{2}$ & $\frac{1}{2}$ & $\frac{1}{2}$ & 1 & $\frac{1}{2}$ & 0 & $\frac{1}{2}$ & $\frac{1}{2}$ \\
\hline 0 & 1 & 0 & 0 & 0 & 0 & 0 & 0 & 0 & 1 & 0 & 0 & 0 & 0 & 0 & 0 \\
\hline
\end{tabular}

Обратим внимание на то, что среди естественных $p$-логик присутствует $p$-логика со связками $\vee^{3}$ и $\wedge^{3}$. Заметим, что $\vee^{3}$ и $\wedge^{3}$ есть обычные $\max \left(\right.$ дизъюнкция) и $\min \left(\right.$ конъюнкция), т.е. $\left.\vee^{3}, \wedge^{3},\right\rceil$ есть $p$-логика, описанная в [3]. В этой же работе показано, что трехзначя логика Лукасевича $\mathbf{Ł}_{3}$ есть дважды $p$-логика, т.е. в нашем обозначении логика со связками $\left.\vee^{3}, \wedge^{3},\right\rceil,\lceil$.

Рассмотрим функциональные свойства полученного класса естественных $p$-логик, а также выясним, какие известные трехзначные логики им соответствуют.

УТВЕРЖДЕНИЕ 1. Естественные $р$-логики со связками $\mathrm{V}^{i}$, $\left.\wedge^{i},\right\rceil u \vee^{i}, \wedge^{i},\lceil$, где $(1 \leq i \leq 18 u i \neq 3)$, функиионально эквивалентны.

Доказательство. Функциональная эквивалентность логик со связками $\left.\vee^{i}, \wedge^{i},\right\rceil$ и $\vee^{i}, \wedge^{i},\lceil$, где $(1 \leq i \leq 18$ и $i \neq 3)$ следует из соотношений (1)-(3):

(1) $i \in\{1,4,7,8,9,10,11,12,13,14,15,16,17,18\}$ 


$$
\begin{aligned}
& \left.\left.\left\lceil p={ }_{D f}\right\rceil\left(p \wedge^{i}\right\rceil\right\rceil p\right), \\
& \rceil p={ }_{D f}\left\lceil\left( p \vee^{i}\lceil\lceil p) ;\right.\right.
\end{aligned}
$$

(2) $i \in\{2,5\}$

$$
\begin{aligned}
& \left.\left.\left.\left\lceil p={ }_{D f}\right\rceil\left(\left(p \vee^{i}\right\rceil p\right) \wedge^{i}\right\rceil\right\rceil p\right), \\
& \rceil p={ }_{D f}\left\lceil\left(\left( p \wedge^{i}\lceil p) \vee^{i}\lceil\lceil p) ;\right.\right.\right.
\end{aligned}
$$

(3) $\left.\left\lceil p={ }_{D f}\right\rceil\left(p \vee^{6}\right\rceil p\right)$,

$$
\rceil p={ }_{D f}\left\lceil\left( p \wedge^{6}\lceil p) .\right.\right.
$$

Утверждение доказано.

Q.E.D.

Таким образом, поскольку логики со связками $\left.\vee^{i}, \wedge^{i},\right\rceil$ и $\vee^{i}, \wedge^{i}$, $\lceil$, где $(1 \leq i \leq 18$ и $i \neq 3)$, функционально эквивалентны, достаточно будет рассмотреть логики со связками $\left.\vee^{i}, \wedge^{i},\right\rceil$.

Далее, нам понадобится следующее утверждение.

УТВЕРЖДЕНИЕ 2. В естественных р-логиках со связками $\left.\vee^{i}, \wedge^{i},\right\rceil$, где $i \in\{1,2,6,8,9,10,12,13,14,15,16,18\}$, выразимо отрицание $\sim$.

Доказательство. В силу ранее доказанного утверждения 1 доказательство утверждения 2 следует из соотношений (1)-(4):

(1) $i \in\{1,2,9,15,16\}$

$\sim p={ }_{D f}\left\lceil p \wedge^{i}(\rceil p \vee^{i} p\right)$

(2) $i \in\{8,10,12,14,18\}$

$\sim p={ }_{D f}\left(p \vee^{i}\lceil p) \wedge^{i}\right\rceil p ;$

(3) $\left.\sim p={ }_{D f}\left(p \vee^{6}\right\rceil p\right) \wedge^{6}\lceil p$;

(4) $\left.\sim p={ }_{D f}\right\rceil p \wedge^{13}\left(\left\lceil p \vee^{13} p\right)\right.$.

Утверждение доказано.

Q.E.D.

Перейдем к рассмотрению взаимоотношений между естественными $p$-логиками.

УТВЕРЖДЕНИЕ 3. Р-логики со связками $\left.\vee^{i}, \wedge^{i},\right\rceil(i \in\{4,5,7\})$ попарно функиионалъно эквивалентны. 
Доказательство. При доказательстве будем учитывать ранее доказанное утверждение 1.

Сначала покажем эквивалентность логик со связками $\left.\vee^{5}, \wedge^{5},\right\rceil$ и $\left.\vee^{7}, \wedge^{7},\right\rceil$. Это верно, в силу следующих соотношений:

(1) $\left.\left.\left.\left.p \vee^{7} q={ }_{D f}\right\rceil\right\rceil p \vee^{5}\right\rceil\right\rceil q$, $p \wedge^{7} q={ }_{D f}\left(p \vee^{5} p\right) \wedge^{5}\left(q \vee^{5} q\right) ;$

(2) $p \vee^{5} q={ }_{D f}\left\lceil\left\lceil p \vee^{7}\lceil\lceil q\right.\right.$, $p \wedge^{5} q={ }_{D f}\left(p \vee^{7} p\right) \wedge^{7}\left(q \vee^{7} q\right)$.

Далее покажем, что логика со связками $\left.\vee^{7}, \wedge^{7},\right\rceil$ функционально эквивалентна логике со связками и $\vee^{4}, \wedge^{4}, 7$.

Это справедливо в силу следующих соотношений:

(1) $\left.\left.\left.\left.p \vee^{7} q={ }_{D f}\right\rceil\right\rceil p \vee^{4}\right\rceil\right\rceil q$, $p \wedge^{7} q={ }_{D f}\left(p \vee^{4} p\right) \wedge^{4}\left(q \vee^{4} q\right) ;$

(2) $\left.\left.p \vee^{4} q={ }_{D f}\left((\rceil q \wedge^{7}\right\rceil\right\rceil p\right) \vee^{7}\left\lceil\lceil q) \vee^{7}\lceil\lceil p\right.$, $\left.\left.\left.p \wedge^{4} q={ }_{D f}((\rceil\rceil q \wedge^{7}\lceil q) \wedge^{7}\right\rceil\right\rceil p\right) \vee^{7}\left(p \wedge^{7} q\right)$.

Таким образом, утверждение доказано.

Q.E.D.

Заметим, что паранепротиворечивая логика $\mathbf{P}^{\mathbf{1}}[10]$ есть логика с исходными связками $\left\lceil\right.$ и $\rightarrow_{7}$. Оказалось, $\mathbf{P}^{\mathbf{1}}$ можно представить в терминах естественных р-логик.

УТВЕРЖДЕНИЕ 4. Логика $\mathbf{P}^{\mathbf{1}}$ есть р-логика со связками $\left.\vee^{7}, \wedge^{7},\right\rceil$.

Доказательство. Покажем, что логика со связками $\left\lceil\right.$ и $\rightarrow_{7}$ функционально эквивалентна логике со связками $\left.\vee^{7}, \wedge^{7},\right\rceil$. Это справедливо, поскольку имеют место следующие соотношения:

(1) $p \vee^{7} q={ }_{D f}\left(\left(p \rightarrow_{7} q\right) \rightarrow_{7} q\right)$, $\left.\left.\left.p \wedge^{7} q={ }_{D f}\right\rceil(\rceil\right\rceil p \rightarrow 7 q\right)$ или $\left.\left.\left.p \wedge^{7} q={ }_{D f}\right\rceil(\rceil p \vee^{7}\right\rceil q\right) ;$ 
(2) $\left.p \rightarrow_{7} q={ }_{D f}(\rceil\left(p \vee^{7} p\right)\right) \vee^{7}\left(q \vee^{7} q\right)$.

Утверждение доказано.

Q.E.D.

В работе [11] исследуется так называемая трехзначная nараполная логика $\mathbf{I}^{\mathbf{1}}$, дуальная к $\mathbf{P}^{\mathbf{1}}$. Это логика с одним выделенным значением и с исходными связками $\rceil$ и $\rightarrow_{5}$. Как и $\mathbf{P}^{1}$, логика $\mathbf{I}^{\mathbf{1}}$ представима в терминах естественных $p$-логик.

УТВЕРЖДЕНИЕ 5. Логика $\mathbf{I}^{\mathbf{1}}$ есть р-логика со свлзками $\bigvee^{5}$, $\left.\wedge^{5},\right\rceil$.

Доказательство. Покажем, что логика со связками $]$ и $\rightarrow_{5}$ функционально эквивалентна логике со связками $\left.\vee^{5}, \wedge^{5},\right\rceil$. Это справедливо, поскольку имеют место следующие соотношения:

(1) $p \vee^{5} q={ }_{D f}\left(\left(p \rightarrow_{5} q\right) \rightarrow_{5} q\right)$,

$$
\left.\left.\left.\left.\left.\left.\left.p \wedge^{5} q={ }_{D f}\right\rceil(\rceil\right\rceil p \rightarrow_{5}\right\rceil q\right) \text { или } p \wedge^{5} q={ }_{D f}\right\rceil(\rceil p \vee^{5}\right\rceil q\right) ;
$$

(2) $\left.p \rightarrow_{5} q={ }_{D f}(\rceil\left(p \vee^{5} p\right)\right) \vee^{5}\left(q \vee^{5} q\right)$.

Утверждение доказано.

Q.E.D.

Таким образом, следствием доказанных утверждений 3-5, является тот факт, что логики $\mathbf{P}^{\mathbf{1}}$ и $\mathbf{I}^{\mathbf{1}}$, ранее рассматриваемые в литературе исключительно как дуальные по отношению друг к другу и совершенно различные, с функциональной точки зрения представляют собой одну и ту же логику.

Заметим, что кроме $\mathbf{P}^{\mathbf{1}}$ и $\mathbf{I}^{\mathbf{1}}$ появилась некоммутативная функционально эквивалентная им логика со связками $\left.\vee^{4}, \wedge^{4},\right\rceil$, которую обозначим посредством $\mathbf{T}^{\mathbf{8}}$.

В статье [9] указано, что логики $\mathbf{P}^{\mathbf{1}}$ и $\mathbf{I}^{\mathbf{1}}$ являются комбинациeй двух изоморфов ${ }^{2}$ классической логики $\mathbf{C}_{\mathbf{2}}$, содержащихся в $\mathbf{B}_{\mathbf{3}}$, однако, поскольку эти логики функционально эквивалентны, справедливо говорить о том, что логика $\mathbf{P}^{\mathbf{1}}\left(\mathbf{I}^{\mathbf{1}}\right)$ содержит два изоморфа классической логики $\mathbf{C}_{\mathbf{2}}$.

\footnotetext{
${ }^{2}$ Адаптируя терминологию Д.А. Бочвара [1] к языку настоящей работы, под трехзначным изоморфом $\mathbf{C}_{2}$ понимается фрагмент трехзначной логики по классу тавтологий, совпадающий с $\mathbf{C}_{2}$. Подробно об изоморфах см. [2].
} 
Далее, рассмотрим другой класс естественных $p$-логик. Для удобства обозначим $p$-логику со связками $\left.\vee^{8}, \wedge^{8},\right\rceil$ как логику $\mathbf{T}^{\mathbf{7}}$. Тогда можем указать целый класс $p$-логик, эквивалентных $\mathbf{T}^{7}$.

УТВЕРЖЖДЕНИЕ 6. Р-логика со связками $\left.\vee^{i}, \wedge^{i},\right\rceil(i \in\{1,2,6$, $10,12,13,18\})$ функционально эквивалентна логике $\mathbf{T}^{\mathbf{7}}$.

Доказательство. Учитывая ранее доказанное утверждение 2 и определение конъюнкции в $p$-логике, при доказательстве эквивалентности соответствующих $p$-логик достаточно показать взаимовыразимость дизъюнкций соответствующих $p$-логик. При доказательстве также будем учитывать следствие из доказанного утверждения 1 , т.е. тот факт, что в $p$-логиках со связками $\left.\vee^{i}, \wedge^{i},\right\rceil$, где $(1 \leq i \leq 18$ и $i \neq 3)$ выразимо отрицание $\lceil$.

(a) Покажем, что $p$-логика со связками $\left.\vee^{i}, \wedge^{i},\right\rceil(i \in\{10,12,13$, $18\})$ и логика $\mathbf{T}^{\mathbf{7}}$ функционально эквивалентны. Это имеет место в силу соотношений:

(1) $\left.\left.p \vee^{10} q={ }_{D f} p \vee^{8}\right\rceil\right\rceil q$, $p \vee^{8} q={ }_{D f}\left(\left(p \vee^{10}\left\lceil\lceil q) \vee^{10}\right\rceil\right\rceil q\right) ;$

(2) $\left.\left.\left.p \vee^{18} q={ }_{D f} p \vee^{8}((\rceil\rceil q \wedge^{8}\right\rceil\right\rceil p\right) \vee^{8}\lceil\lceil q)$, $\left.p \vee^{8} q={ }_{D f}\left(p \vee^{18}\left\lceil\lceil q) \vee^{18}\right\rceil\right\rceil\right) ;$

(3) $p \vee^{12} q={ }_{D f}\left(\left(p \vee^{8} q\right) \wedge^{8}(\rceil q \vee^{18}\lceil\lceil p)) \vee^{8}\lceil\lceil q\right.$, $\left.\left.p \vee^{8} q={ }_{D f}\left(p \vee^{12} q\right) \vee^{12}\right\rceil\right\rceil q$;

(4) $p \vee^{12} q={ }_{D f}\left\lceil\left\lceil q \vee^{13}\left(\left(q \vee^{13} p\right) \wedge^{13}(\rceil q \vee^{13}\lceil\lceil p))\right.\right.\right.$, $p \vee^{13} q={ }_{D f}\left(q \vee^{12} p\right) \vee^{12}\left(p \vee^{12} q\right)$.

(б) Далее покажем, что $p$-логика со связками $\left.\vee^{i}, \wedge^{i},\right\rceil(i \in\{1,2$, $6\})$ и логика $\mathbf{T}^{\mathbf{7}}$ функционально эквивалентны. Это имеет место в силу соотношений:

(1) $\left.\left.\left.p \vee^{1} q={ }_{D f}(\rceil\right\rceil p \wedge^{2}\right\rceil q\right) \vee^{2}\left(p \vee^{2} q\right)$, $p \vee^{2} q={ }_{D f}\left(\left\lceil\left\lceil p \vee^{1}\right\rceil\right\rceil q\right) \wedge^{1}\left(p \vee^{1} q\right) ;$ 
(2) $p \vee^{2} q={ }_{D f} q \vee^{6}\left(p \vee^{6} q\right)$, $p \vee^{6} q={ }_{D f}\left(\left(q \vee^{2} p\right) \wedge^{2}(\rceil q \vee^{2}\lceil\lceil q)) \vee^{2}\lceil\lceil p ;\right.$

(3) $\left.\left.\left.\left.p \vee^{8} q={ }_{D f} p \vee^{6}\left(\left(\left\lceil q \wedge^{6}\right\rceil\right\rceil p\right) \vee^{6}(\rceil p \wedge^{6}\right\rceil\right\rceil q\right)\right)$, $p \vee^{6} q={ }_{D f}\left(\left(p \vee^{8}\right\rceil q\right) \vee^{8}\lceil p) \wedge^{8}\left(\left\lceil\left\lceil q \vee^{8}\lceil\lceil p)\right.\right.\right.$.

Очевидно, доказательство утверждения 6 следует из доказанных положений (а) и (б).

Q.E.D.

Далее,

УТВЕРЖКДЕНИЕ 7. Р-логика со связками $\left.\vee^{i}, \wedge^{i},\right\rceil(i \in\{9,14$, 16\}) есть трехзначная логика Лукасевича $\mathbf{E}_{\mathbf{3}}$.

\section{Доказательство.}

(a) Сначала покажем, что $p$-логики со связками $\left.\vee^{9}, \wedge^{9},\right\rceil, \vee^{14}$, $\left.\wedge^{14},\right\rceil$ и $\left.\vee^{16}, \wedge^{16},\right\rceil$ попарно функционально эквивалентны. Учитывая определение конъюнкции в $p$-логике и то, что в указанных $p$-логиках выразимы $\sim$ и $\lceil$ (утверждения 1,2 , доказательство положения (а) следует из соотношений (1) и (2):

(1) $\left.p \vee^{9} q={ }_{D f}\left(p \vee^{16} q\right) \vee^{16}(\rceil\right\rceil q \wedge^{16}\lceil p)$, $p \vee^{16} q={ }_{D f}\left(\sim q \wedge^{9} q\right) \vee^{9}\left(p \vee^{9} q\right)$;

(2) $\left.p \vee^{16} q={ }_{D f}\left(\left(p \vee^{14} q\right) \vee^{14}(\rceil\right\rceil p \wedge^{14}\lceil p)\right) \vee^{14} q$, $p \vee^{14} q={ }_{D f}\left(\left(p \vee^{16}\left\lceil\lceil q) \vee^{16}\left(q \vee^{16}\lceil\lceil p)) \wedge^{16}\left(\left(\left\lceil\left\lceil q \wedge^{16}\right\rceil\right\rceil p\right) \vee^{16}\right.\right.\right.\right.\right.$ (\rceil $\left.q \vee^{16}\lceil p)\right)$.

(б) Докажем, что, например, $p$-логика со связками $\left.\vee^{16}, \wedge^{16},\right\rceil$ есть $\mathbf{E}_{\mathbf{3}}$.

Это справедливо, с одной стороны, в силу функциональной предполноты $\mathbf{L}_{3}$, с другой стороны, в силу того, что посредством связок $\left.\vee^{14}, \wedge^{14},\right\rceil$ определима импликация логики Лукасевича (в нашем обозначении $\left.\rightarrow_{3}\right)^{3}$.

$$
\left.p \rightarrow_{3} q=_{D f}\left(\left(\sim p \wedge^{14} q\right) \vee^{14}(\rceil\right\rceil q \vee^{14}\lceil p)\right) .
$$

\footnotetext{
${ }^{3}$ Для доказательства достаточно определить импликацию Лукасевича, поскольку известно, что в логике со связками $\left.\vee^{14}, \wedge^{14},\right\rceil$ выразимо отрицание Лукасевича $\sim$ (доказанное ранее утверждение 2 ).
} 
Таким образом, утверждение 7 доказано.

Q.E.D.

УТВЕРЖДЕНИЕ 8. Р-логики со связками $\left.\vee^{11}, \wedge^{11},\right\rceil u \vee^{17}$, $\left.\wedge^{17},\right\rceil$ функционально эквивалентны.

Доказательство. Доказательство утверждения следует из соотношений:

(1) $p \vee^{11} q={ }_{D f} p \vee^{17}\left(q \vee^{17} p\right)$, $p \wedge^{11} q={ }_{D f} q \wedge^{17}\left(p \wedge^{17} q\right) ;$

(2) $p \vee^{17} q={ }_{D f}\left(\left(p \vee^{11} q\right) \wedge^{11} q\right) \vee^{11} p$ $p \wedge^{17} q={ }_{D f}\left(\left(p \wedge^{11} q\right) \vee^{11} q\right) \wedge^{11} p$.

Утверждение доказано.

Q.E.D.

Для удобства $p$-логику со связками $\left.\vee^{11}, \wedge^{11},\right\rceil$ обозначим $\mathbf{T}^{\mathbf{5}}$. $P$-логику со связками $\left.\vee^{15}, \wedge^{15},\right\rceil$ обозначим $\mathbf{T}^{\mathbf{6}}$.

Таким образом, класс естественных $p$-логик разбивается на пять подклассов: это $p$-логики, функционально эквивалентные логике Лукасевича $\mathbf{E}_{\mathbf{3}}, p$-логики, функционально эквивалентные логике Сетте $\mathbf{P}^{\mathbf{1}}, p$-логики, функционально эквивалентные логикам $\mathbf{T}^{\mathbf{5}}, \mathbf{T}^{\mathbf{6}}, \mathbf{T}^{\mathbf{7}}$ соответственно. Связки конъюнкции и дизъюнкции в $\mathbf{T}^{\mathbf{7}}$ и $\mathbf{T}^{\mathbf{6}}$ являются некоммутативными. Интересно, что для логики $\mathbf{T}^{\mathbf{5}}$ получили два эквивалентных построения с некоммутативными связками $\left(\vee^{17}, \wedge^{17}\right)$ и коммутативными $\left(\vee^{11}\right.$, $\left.\wedge^{11}\right)$.

Заметим, что таблицы для связок $\vee^{11}, \wedge^{11}$ логики $\mathbf{T}^{\mathbf{5}}$ встречаются в работе [5], где приводится классификация трехзначных логик значения, исходя из свойств соответствующих им универсальных алгебр. Логика со связками $\vee^{11}, \wedge^{11}$ относится к классу слабых исчерпывающих логик значения.

В работе [7] описаны 11 предполных классов логики Бочвара $\mathbf{B}_{3}$ и логика $\mathbf{T}^{\mathbf{6}}$ является одним из них. Заметим, с этой точки зрения, логика $\mathbf{P}^{1}$ есть класс всех внешних функций. Покажем, что это так.

В работе [8] В.И. Шестаков определяет штрих Шеффера $\gamma$ для внешних связок $\mathbf{B}_{\mathbf{3}}$ 


\begin{tabular}{|c|ccc|}
\hline$\gamma$ & 1 & $\frac{1}{2}$ & 0 \\
\hline 1 & 0 & 0 & 0 \\
$\frac{1}{2}$ & 0 & 0 & 1 \\
0 & 0 & 1 & 1 \\
\hline
\end{tabular}

Это множество связок он обозначает как $\mathbf{b}_{\mathbf{1}}$. Тогда докажем следующее утверждение:

УТВЕРЖДЕНИЕ 9. Логика $\mathbf{P}^{\mathbf{1}}$ есть $\mathbf{6}_{\mathbf{1}}$.

Доказательство. Докажем функциональную эквивалентность $\mathbf{P}^{1}$ и $\mathbf{b}_{1}$.

Очевидно, связки $\mathbf{P}^{\mathbf{1}}$ есть внешние связки $\mathbf{b}_{\mathbf{1}}$, т.е. они определимы посредством штриха Шеффера $\gamma$.

Далее посредством связок логики $\mathbf{P}^{1}$ определим штрих Шеффера $\gamma$. Согласно ранее доказанному утверждению 4 , логика $\mathbf{P}^{1}$ есть логика со связками $\vee^{7}, \wedge^{7},\left\lceil\right.$, также в $\mathbf{P}^{\mathbf{1}}$ выразимо отрицание 7 (доказанное утверждение 1). Тогда определить штрих Шеффера можно следующим образом:

$$
p \gamma q={ }_{D f}(\rceil p \wedge^{7}\lceil q) \vee^{7}\left(\left\lceil p \wedge^{7}\right\rceil q\right) .
$$

Утверждение 9 доказано.

Q.E.D.

Здесь стоит отметить, что класс внешних связок был впервые выделен в 1938 г. Д.А. Бочваром [1]. Как только что указывалось, специально этот класс рассмотрен в [8]. Здесь же представлены нормальные формы для Б $\mathbf{1}$. Далее, в работе В.К. Финна [7] описаны все предполные классы $\mathbf{b}_{\mathbf{1}}-$ семь классов.

При изучении функциональных свойств $p$-логик относительно логики $\mathbf{P}^{\mathbf{1}}$ справедливо следующее утверждение:

УТВЕРЖДЕНИЕ 10. $\mathbf{P}^{\mathbf{1}}$ функционально вложима в некоторую p-логику со связками $\vee, \wedge$,$\rceil , если р-логика обладает следую-$ щими свойствами:

(1) р-логика функиионально эквивалентна дважды р-логике (т.е. в р-логике выразимо отрицание Г); 
(2) связки $\vee, \wedge$ являются C-расширяющими, т.е. сохраняются классические значения, когда аргументы принимают значения из множества $\{0,1\}$.

Доказательство. Для доказательства утверждения покажем, что посредством связок $\vee, \wedge$,$\rceil выразимы связки логики \mathbf{P}^{\mathbf{1}}$. Очевидно, достаточно показать, что выразимы $\vee^{7}, \wedge^{7}$.

$$
\begin{aligned}
& \left.\left.\left.\left.p \vee^{7} q={ }_{D f}\right\rceil\right\rceil p \vee\right\rceil\right\rceil q, \\
& p \wedge^{7} q={ }_{D f}\lceil\lceil p \wedge\lceil\lceil q .
\end{aligned}
$$

Утверждение 10 доказано.

Q.E.D.

Очевидными следствиями утверждения 10 являются следующие: (1) $\mathbf{P}^{\mathbf{1}} \subset \mathbf{L}_{\mathbf{3}}$; (2) $\mathbf{P}^{\mathbf{1}} \subset \mathbf{T}^{\mathbf{7}}$; (3) $\mathbf{P}^{\mathbf{1}} \subset \mathbf{T}^{\mathbf{6}}$; (4) $\mathbf{P}^{\mathbf{1}} \subset \mathbf{T}^{\mathbf{5}}$.

Далее покажем, что логики $\mathbf{T}^{\mathbf{5}}$ и $\mathbf{T}^{\mathbf{6}}$ функционально независимы, т.е. $\mathbf{T}^{\mathbf{5}} \not \subset \mathbf{T}^{6}$ и $\mathbf{T}^{\mathbf{6}} \not \subset \mathbf{T}^{5}$.

$\mathbf{T}^{\mathbf{6}} \not \subset \mathbf{T}^{\mathbf{5}}$, так как согласно утверждению 2 в $\mathbf{T}^{\mathbf{6}}$ выразимо отрицание $\sim$, в то время как посредством связок $\mathbf{T}^{\mathbf{5}}$ отрицание $\sim$ определить невозможно ${ }^{4}$. С другой стороны, как было ранее упомянуто, логика $\mathbf{T}^{\mathbf{6}}$ есть предполный в $\mathbf{B}_{\mathbf{3}}$ класс функций. Из построения нормальных форм $(I-J$-с.д.н.ф. $)$ для логики $\mathbf{B}_{\mathbf{3}}[6]$ следует, что, например, дизъюнкция $\vee^{11}$ логики $\mathbf{T}^{\mathbf{5}}$ не определима в $\mathbf{B}_{\mathbf{3}}$, а значит, ее нельзя определить и с помощью связок $\mathbf{T}^{6}$. Таким образом, $\mathbf{T}^{\mathbf{5}} \not \subset \mathbf{T}^{\mathbf{6}}$.

УТВЕРЖДЕНИЕ 11. Логика $\mathbf{T}^{\mathbf{5}}$ функционально вложима в логику $\mathbf{T}^{\mathbf{7}}$.

Доказательство. Для доказательства достаточно посредством связок $\left.\vee^{1}, \wedge^{1},\right\rceil$ определить дизъюнкцию $\vee^{11}$. Это можно сделать, например, так:

$$
p \vee^{11} q={ }_{D f}\left(p \vee^{1} q\right) \vee^{1} p .
$$

Утверждение 11 доказано.

Q.E.D.

\footnotetext{
${ }^{4}$ Свойства $\vee, \wedge$ логики $\mathbf{T}^{\mathbf{5}}$ таковы, что (1) они принимают значение $\frac{1}{2}$ только в одном случае - при значении аргументов $\frac{1}{2}$; (2) $x \vee x=x$ и $x \wedge x=x$. Таким образом, посредством связок $\vee, \wedge,\rceil,\lceil$ определить $\sim$ нельзя.
} 
УТВЕРЖДЕНИЕ 12. Логика $\mathbf{T}^{\mathbf{6}}$ функиионально вложима в логику $\mathbf{T}^{\mathbf{7}}$.

Доказательство. Для доказательства достаточно посредством связок $\left.\vee^{1}, \wedge^{1},\right\rceil$ определить дизъюнкцию $\vee^{15}$. Это можно сделать, например, так:

$$
\left.\left.\left.p \vee^{15} q=_{D f}(\rceil\right\rceil p \wedge^{1}\right\rceil q\right) \vee^{1} q
$$

Утверждение 12 доказано.

Q.E.D.

С другой стороны, свойства связок логики $\mathbf{T}^{\mathbf{7}}$ таковы, что $\mathbf{T}^{\mathbf{7}}$ не вложима ни в $\mathbf{T}^{\mathbf{5}}$, ни в $\mathbf{T}^{\mathbf{6}}$.

$\mathbf{T}^{\mathbf{7}} \not \subset \mathbf{T}^{\mathbf{5}}$, так как согласно утверждению 2 в $\mathbf{T}^{\mathbf{7}}$ выразимо отрицание $\sim$, в то время как было ранее указано, посредством связок $\mathbf{T}^{\mathbf{5}}$ отрицание $\sim$ определить невозможно.

$\mathbf{T}^{\mathbf{7}} \not \subset \mathbf{T}^{\mathbf{6}}$, поскольку $\mathbf{T}^{\mathbf{6}} \subset \mathbf{B}_{\mathbf{3}}$, и из построения нормальных форм (I-J-с.д.н.ф.) для логики $\mathbf{B}_{\mathbf{3}}[6]$ следует, что, например, дизъюнкция $\vee^{1}$ логики $\mathbf{T}^{\mathbf{7}}$ не определима в $\mathbf{B}_{\mathbf{3}}$, а значит, ее нельзя определить и с помощью связок $\mathbf{T}^{\mathbf{6}}$.

Далее, в силу функциональной предполноты логики $\mathbf{E}_{3}$ верно, что $\mathbf{T}^{\mathbf{7}} \subset \mathbf{E}_{\mathbf{3}}$. Однако $\mathbf{E}_{\mathbf{3}}$ не вложима в $\mathbf{T}^{\mathbf{7}}$, в противном случае, в $\mathbf{T}^{\mathbf{7}}$ была бы выразима, например, сильная регулярная дизъюнкция $\vee$. Возьмем в качестве исходных связок $\mathbf{T}^{\mathbf{7}}$ связки $\left.\vee^{1}, \wedge^{1},\right\rceil$.

Имеем: $0 \vee \frac{1}{2}=\frac{1}{2} \vee 0=\frac{1}{2} \vee \frac{1}{2}=\frac{1}{2}$. Свойства связок $\vee^{1}, \wedge^{1}$ таковы, что невозможно определить такую связку $\vee^{\mathrm{x}}$, чтобы $0 \vee^{\mathrm{x}} \frac{1}{2}=\frac{1}{2} \vee^{\mathrm{x}} 0=\frac{1}{2}$ или $1 \bigvee^{\mathrm{x}} \frac{1}{2}=\frac{1}{2} \vee^{\mathrm{x}} 1=\frac{1}{2}$. Подобные рассуждения дают основания говорить, что $\mathbf{E}_{\mathbf{3}} \not \subset \mathbf{T}^{\mathbf{7}}$.

В результате, класс естественных $p$-логик образует решетку по отношению функционального вложения одной логики в другую, и в качестве элементов решетки выступают трехзначные логики - логика Лукасевича $\mathbf{L}_{\mathbf{3}}$, логика Сетте $\mathbf{P}^{\mathbf{1}}$, а также новые логики $\mathbf{T}^{5}, \mathbf{T}^{6}, \mathbf{T}^{7}$ : 


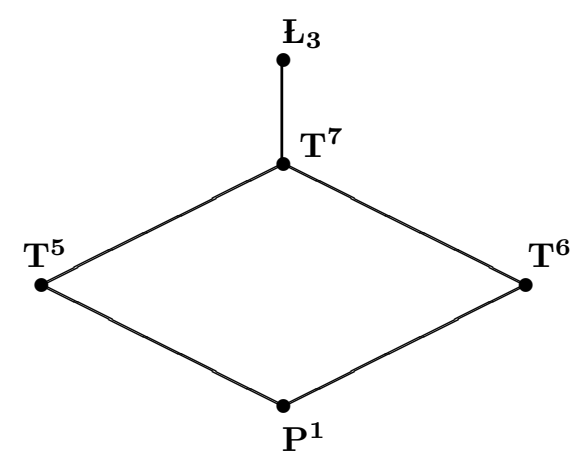

Рис.1. Решетка естественных $p$-логик

\section{Литература}

[1] Бочвар Д.А. Об одном трехзначном исчислении и его применении к анализу парадоксов классического расширенного функциональого исчисления // Математический сборник. 1938. Т. 4. № 2. С. 287-308.

[2] Девяткин Л.Ю. Многозначные изоморфы классической пропозициональной логики. Дис. ... канд. филос. наук. М., 2008.

[3] Карпенко A.C. Р-логики // Современная логика: проблемы теории, истории и применения в науке. (Материалы Х Общероссийской научной конференции, 26-28 июня 2008 г., Санкт-Петербург.) СПб., 2008. С. 278-280.

[4] Томова Н.E. Импликативные расширения регулярных логик Клини // Логические исследования. Вып. 16. М., 2010. С. 233-258.

[5] Финн B.K., Аншаков О.М., Григолия Р.Ш., Забежайло М.И. Многозначные логики как фрагменты формализованной семантики // Семиотика и информатика. 1980. Вып. 15. С. 27-60.

[6] Финн B.K. Аксиоматизация некоторых трехзначных исчислений высказываний и их алгебр // Философия в современном мире. Философия и логика. М.: Наука, 1974. C. $398-438$.

[7] Финн В.К. О критерии функциональной полноты для В3 // Исследования по формализованным языкам и неклассическим логикам. М.: Наука, 1974. С. 194199.

[8] Шестаков В.И. Об одном фрагменте исчисления Д.А. Бочвара // Информационные вопросы семиотики, лингвистики и автоматического перевода. ВИНИТИ. Вып. 1. М., 1971.

[9] Karpenko A.S. A maximal paraconsistent logic: The combination of two threevalued isomorphs of classical propositional logic // Fronties of Paraconsistent Logic. Baldock: Research Studies Press, 2000. P. 181-187.

[10] Sette A.M. On propositional calculus P1 // Mathematica Japonica. 1973. Vol. 18. № 3. P. $173-180$.

[11] Sette A.M. and Carnielli W.A. Maximal weakly-intuitionistic logics // Studia Logica. 1995. Vol. 55. P. 181-203. 